coloproctology $2011 \cdot 33: 251$

DOI 10.1007/s00053-011-0202-3

Online publiziert: 19. Juni 2011

(c) Springer-Verlag 2011

\author{
A. Herold \\ End- und Dickdarm-Zentrum Mannheim
}

\title{
Sakralnervenstimulation bei hartnäckiger Obstipation
}

duziert wurde die auf der Toilette verbrachte Zeit (10,5 auf 5,7 min; $p=0,001)$, Pressen (75-46\% erfolgreiche Entleerungen; $\mathrm{p}<0,001)$, das Gefühl unvollständiger Entleerung (71,5-46\% erfolgreiche Entleerungen; $p<0,001)$ und die subjektive Einstufung des abdominellen Schmerzes sowie Blähungen ( $\mathrm{p}<0,001)$. Der ClevelandClinic-Obstipations-Score (o: keine bis 30: schwere Obstipation) nahm von 18 auf $10 \mathrm{ab}(\mathrm{p}<\mathrm{o}, 001)$. Der Score (o: schwer bis 100: keine Symptome) der visuellen Analogskala (VAS) stieg von 8 auf 66 $(p<0,001)$. Patienten mit Slow- und Normal-Transit profitierten. Die Lebensqualität verbesserte sich signifikant. Die Kolontransitzeit normalisierte sich bei 50\% der Patienten mit einem Slow-Transit zu Studienbeginn ( $\mathrm{p}=\mathrm{o}, \mathrm{O14})$.

Schlussfolgerung. Die SNS ist effektiv bei der Behandlung idiopathischer SlowTransit- und Normal-Transit-Obstipation, bei denen eine konservative Behandlung erfolglos war.

\section{Kommentar}

Seit über 10 Jahren wird die Sakralnervenstimulation (SNS) mit großem Erfolg bei analer Inkontinenz eingesetzt. Hat man in der Anfangszeit immer vermutet, dass der wesentliche Effekt durch eine direkte Stimulation des Nervus pudendus zu sehen ist, konnte dies jedoch mit objektiven Messmethoden nicht belegt werden. So steigt z. B. der anale Ruhedruck, wie anfänglich vermutet, nicht an. Der wesentliche Therapieeffekt ist somit in einer Modulation des gesamten Zusammenspiels zwischen zentralem Nervensystem, peripherem Nervensystem, Rektumreservoir und auch dem Analsphinkter zu sehen.
In urologischen und chirurgischen Studien zur Harn- bzw. Stuhlinkontinenz hat man als Nebeneffekt bei einigen Patienten eine Verbesserung einer gleichzeitig bestehenden chronischen Obstipation gesehen. In diesen Studien hatte man diesen Therapieeffekt jedoch nicht gesondert evaluiert.

Vor diesem Hintergrund wurde nun obige Multizenterstudie zur Therapie der chronischen Obstipation durchgeführt. Kann man sich einen Effekt bei Entleerungsstörung durchaus vorstellen, so ist der Therapieerfolg bei Slow-TransitObstipation für den allgemeinen Leser überraschend. Die Ergebnisse der o. g. Studie sind für beide Formen der chronischen Obstipation sehr gut. Es ist besonders zu erwähnen, dass die Stuhlfrequenz signifikant angestiegen ist und gleichzeitig die Zeit der Stuhlentleerung (Zeit auf der Toilette) signifikant verkürzt wurde. Als weiterer Benefit wurde eine Verbesserung der abdominellen Beschwerden, insbesondere der Blähungen, berichtet.

Um den Wirkmechanismus der SNS bei chronischer Obstipation zu erforschen, sind jedoch neben weiteren psychologischen und pathophysiologischen Studien auch prinzipiell weitere Untersuchungen an größeren Patientenkollektiven erforderlich. Für Patienten mit chronischer Obstipation stellt diese Methode möglicherweise jedoch eine durchaus überlegenswerte neue Therapieoption dar.

\section{Korrespondenzadresse \\ Prof. Dr. A. Herold}

End- und Dickdarm-Zentrum Mannheim

Bismarckplatz 1, 68165 Mannheim

a.herold@heroldhomeline.de

Interessenkonflikt. Der korrespondierende Autor gibt an, dass kein Interessenkonflikt besteht.
Anzahl der Tage pro Woche mit Entleerung stieg von 2,3 auf 4,8 ( $\mathrm{p}<0,001)$. Re- 